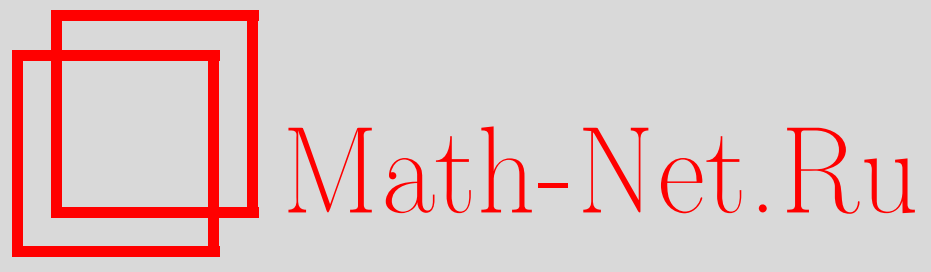

Д. В. Егоров, QR-подмногообразия и римановы метрики с группой голономии $G_{2}$, Матем. заметки, 2011, том 90 , выпуск 5, 781-784

DOI: https://doi.org/10.4213/mzm9269

Использование Общероссийского математического портала Math-Net.Ru подразумевает, что вы прочитали и согласны с пользовательским соглашением http://www . mathnet.ru/rus/agreement

Параметры загрузки:

IP: 3.91 .87 .62

26 апреля 2023 г., 13:52:11

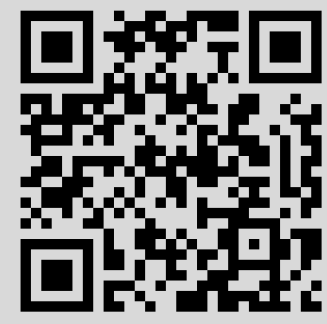




\section{QR-подмногообразия и римановы метрики с группой голономии $G_{2}$}

\section{Д. В. Егоров}

\section{1. Введение}

Изучению многообразий с группой голономии $G_{2}$ препятствует недостаток явных примеров. Первые примеры полных римановых метрик с группой голономии $G_{2}$ были построены Брайантом и Саламоном в [1], а первые компактные примеры - Джойсом [2]. Позже Ковалев построил еще несколько семейств компактных примеров [3], [4]. Заметим, что метрики, построенные в работах [2]-[4] не задаются явными формулами.

Нехватка примеров является следствием того, что $G_{2}$-многообразия, вообще говоря, не являются алгебраическими в широком смысле данного термина.

В данной работе мы делаем попытку частично объяснить этот факт, выдвигая гипотезу, что в общем случае $G_{2}$-многообразия являются QR-подмногообразиями гиперкэлеровых многообразий. Примерами QR-подмногообразий являются вещественные гиперповерхности гиперкэлеровых многообразий.

1.1. $G_{2}$-структура. Определим 3 -форму $\Omega_{0}$ на $\mathbb{R}^{7}$ по следующей формуле:

$$
\Omega_{0}=x^{127}+x^{136}+x^{145}+x^{235}-x^{246}+x^{347}+x^{567},
$$

где $x^{i j k}=x^{i} \wedge x^{j} \wedge x^{k}$. Подгруппа $G L(7, \mathbb{R})$, сохраняющая ориентацию и форму $\Omega_{0}$, называется группой $G_{2}$.

Пусть $M$ - ориентируемое замкнутое многообразие размерности 7 . На многообразии $M$ задана $G_{2}$-структура, если существует глобальная 3 -форма $\Omega$ такая, что всюду на $M$ она поточечно совпадает с $\Omega_{0}$ при подходящем выборе координат.

$G_{2}$-структура задает ориентацию и риманову метрику $g_{\Omega}$ по следующей формуле:

$$
\left(i_{\xi} \Omega\right) \wedge\left(i_{\eta} \Omega\right) \wedge \Omega=-6 g_{\Omega}(\xi, \eta) \operatorname{Vol}_{\Omega} .
$$

1.2. Векторные произведения. В данном разделе мы основываемся на работе [5]. Пусть $M$ является многообразием с $G_{2}$-структурой. Векторное произведение на $M$ - это по определению кососимметрическое полилинейное гладкое отображение $P: T M \times T M \rightarrow T M$, следующим образом согласованное с метрикой $g$ :

$$
\begin{gathered}
g\left(P\left(e_{1}, e_{2}\right), e_{i}\right)=0, \quad i=1,2, \\
\left\|P\left(e_{1}, e_{2}\right)\right\|^{2}=\left\|e_{1}\right\|^{2}\left\|e_{2}\right\|^{2}-g\left(e_{1}, e_{2}\right)^{2}, \quad\|e\|^{2}=g(e, e) .
\end{gathered}
$$

Будем также обозначать $P\left(e_{1}, e_{2}\right)$ через $e_{1} \times e_{2}$.

Векторное произведение однозначно задается 3-формой $\Omega$ :

$$
\Omega\left(e_{1}, e_{2}, e_{3}\right)=g\left(P\left(e_{1}, e_{2}\right), e_{3}\right) .
$$

Обратно, по векторному произведению мы можем задать метрику $g$, используя следующее тождество:

$$
P\left(e_{1}, P\left(e_{1}, e_{2}\right)\right)=-\left\|e_{1}\right\|^{2} e_{2}+g\left(e_{1}, e_{2}\right) e_{1} .
$$

Используя (4), по векторному произведению и метрике можно восстановить 3 -форму $\Omega$. Таким образом, задание векторного произведения эквивалентно заданию $G_{2}$-структуры.

Известно, что если $\nabla P=0$ относительно метрической связности, то группа голономии $M$ является подгруппой $G_{2}$ и совпадает с ней при условии, что $\pi_{1}(M)$ является конечной группой [2].

Работа выполнена при поддержке Российского фонда фундаментальных исследований (грант № 09-01-00598-а) и программ Президента РФ "Ведущие научные школы” (грант № НШ-7256.2010.1) и "Молодые кандидаты" (грант № MK-842.2011.1). 
1.3. QR-подмногообразия. Гиперкэлерово многообразие - это по определению ориентируемое $4 n$-мерное риманово многообразие, группа голономии которого содержится в $S p(n)$.

Подмногообразие $M$ гиперкэлерова многообразия $\bar{M}$ называется QR-подмногообразием [6], если нормальное расслоение раскладывается в прямую сумму, $N M=\nu \oplus \nu^{\perp}$ со следующими свойствами:

$$
J_{i} \nu \subset \nu, \quad J_{i} \nu^{\perp} \subset T M,
$$

где $J_{i}$ являются комплексными структурами многообразия $\bar{M}$.

Везде далее мы будем рассматривать QR-подмногообразия с $\operatorname{dim} \nu^{\perp}=1$. Мы будем называть их QR-подмногообразиями гиперповерхностного типа.

\section{2. Основной результат}

Теорема 1. Ориентируемое многообразие $M$ вещественной размерности 7, являющееся QR-подмногообразием гиперповерхностного типа гиперкэлерова многообразия $\bar{M}$, обладает $G_{2}$-структурой, совместной с индуцированной метрикой.

ДокАЗАТЕльство. Построим векторное произведение, совместное с индуцированной метрикой.

Обозначим через $n$ единичное нормальное векторное поле, лежащее в $\nu^{\perp} \subset N M$. Из определения QR-подмногообразия (6) следует, что $\xi_{i}=J_{i} n \in T M$ являются невырожденными векторными полями на $M$. Это согласуется с работой [7], из которой следует, что на многообразии с $G_{2}$-структурой существует 3 невырожденных векторных поля.

Пусть $\xi_{i}$ являются единичными взаимно ортогональными относительно индуцированной метрики векторами всюду на $M$. Дополним локально $\xi_{i}$ до ортонормированного базиса и недостающие вектора обозначим через $\xi_{\alpha}$, т.е. греческими индексами.

Зададим векторное произведение $P$ следующими формулами:

$$
\begin{aligned}
P\left(\xi_{i}, \xi_{j}\right) & =\xi_{k}, \quad(i j k) \in(123), \\
P\left(\xi_{i}, \xi_{\alpha}\right) & =J_{i}\left(\xi_{\alpha}\right), \\
P\left(\xi_{\alpha}, J_{i}\left(\xi_{\alpha}\right)\right) & =\xi_{i} .
\end{aligned}
$$

Из определения QR-подмногообразия гиперповерхностного типа следует, что для любых $\xi_{\alpha}, \xi_{\beta}$ существует комплексная структура $J_{i}$ такая, что $J_{i} \xi_{\alpha}=\xi_{\beta}$. Таким образом, уравнения (7)-(9) задают векторное произведение на всех базисных векторах.

Очевидно $P$ удовлетворяет тождеству (5) для всех базисных векторов и, следовательно, $P$ совместимо с индуцированной метрикой.

Поставим себе задачу найти условия, при которых введенное векторное произведение является параллельным.

Обозначим через $\bar{\nabla}$ и $\nabla$ связность, согласованную с метрикой $\bar{M}$ и $M$ соответственно.

УтвеРЖДЕНИЕ 1. Имеем

$$
\begin{aligned}
\nabla \xi_{i} & =J_{i}(\bar{\nabla} n)-b\left(\xi_{i}\right), \\
\left(\nabla J_{i}\right)\left(\xi_{\alpha}\right) & =J_{i} \circ b\left(\xi_{\alpha}\right)-b \circ J_{i}\left(\xi_{\alpha}\right) .
\end{aligned}
$$

ДокАЗАТЕЛьство. По формуле Гаусса

$$
\bar{\nabla} \xi_{i}=\nabla \xi_{i}+b\left(\xi_{i}\right),
$$

где $b\left(\xi_{i}\right)=b\left(\xi_{i}, \cdot\right)$, и $b-$ это вторая фундаментальная форма.

$\mathrm{C}$ другой стороны, из определения гиперкэлерова многообразия следует, что

$$
\bar{\nabla} \xi_{i}=\bar{\nabla} J_{i}(n)=\left(\bar{\nabla} J_{i}\right)(n)+J_{i}(\bar{\nabla} n) .
$$

Из (12) и (13) получаем искомую формулу (10). 
Аналогично по формуле Гаусса

$$
\bar{\nabla}\left(J_{i} \xi_{\alpha}\right)=\nabla\left(J_{i}\left(\xi_{\alpha}\right)\right)+b\left(J_{i}\left(\xi_{\alpha}\right)\right)=\left(\nabla J_{i}\right)\left(\xi_{\alpha}\right)+J_{i}\left(\nabla \xi_{\alpha}\right)+b\left(J_{i}\left(\xi_{\alpha}\right)\right) .
$$

С другой стороны,

$$
\bar{\nabla}\left(J_{i} \xi_{\alpha}\right)=\left(\bar{\nabla} J_{i}\right)\left(\xi_{\alpha}\right)+J_{i}\left(\bar{\nabla} \xi_{\alpha}\right) .
$$

Из (14) и (15) получаем формулу (11).

Далее мы выпишем компоненты тензора $\nabla P$. Введем обозначения для следующих тензоров:

$$
X_{i}(\xi)=J_{i}\left(\bar{\nabla}_{\xi} n\right)-b\left(J_{i} n, \xi\right), \quad Y_{i}(\xi, \eta)=J_{i} b(\xi, \eta)-b\left(\xi, J_{i} \eta\right) .
$$

УТВеРЖДЕНИЕ 2. Имеем

$$
\begin{aligned}
& (\nabla P)\left(\xi_{i}, \xi_{j}\right)=X_{k}-X_{i} \times \xi_{j}-\xi_{i} \times X_{j}, \\
& (\nabla P)\left(\xi_{i}, \xi_{\alpha}\right)=Y_{i}\left(\xi_{\alpha}\right)-X_{i} \times \xi_{\alpha}, \\
& (\nabla P)\left(\xi_{i}, \xi_{\alpha}\right)=Y_{i}\left(\xi_{\alpha}\right)-X_{i} \times \xi_{\alpha} .
\end{aligned}
$$

ДокАзАтельство. Докажем формулу (16). Продифференцируем (7):

$$
(\nabla P)\left(\xi_{i}, \xi_{j}\right)=\nabla \xi_{k}-P\left(\nabla \xi_{i}, \xi_{j}\right)-P\left(\xi_{i}, \nabla \xi_{j}\right) .
$$

Подставив (10) в (19) и использовав (7), получим формулу (16).

Аналогично дифференцируя формулы (8) и (9) с использованием определения векторного произведения, получим формулы (17) и (18).

Приравнивая к нулю (16)-(18), мы сформулируем следующую теорему. При этом заметим, что выражения (17) и (18) эквивалентны.

Теорема 2. Пусть $M$ - ориентируемое многообразие вещественной размерности 7, являющееся QR-подмногообразием гиперповерхностного типа гиперкэлерова многообразия $\bar{M}$. Тогда $M$ обладает группой голономии, содержащейся в $G_{2}$, при выполнении следующих уравнений:

$$
\begin{aligned}
X_{k}(\xi)-X_{i}(\xi) \times \xi_{j}-\xi_{i} \times X_{j}(\xi) & =0, \\
Y_{i}(\xi, \eta)-X_{i}(\xi) \times \eta & =0
\end{aligned}
$$

для любых $\xi, \eta, J_{i} \eta \in \Gamma(T M), i=1,2,3$.

ПримеР. Простейшими примерами компактных QR-подмногообразий с группой голономии, содержащейся в $G_{2}$, служат вполне геодезические QR-подмногообразия - гиперповерхности. Например, вложение плоских торов: $T^{7} \hookrightarrow T^{8}$ и $T^{3} \times K 3 \hookrightarrow T^{4} \times K 3$.

\section{3. Гипотеза}

Как мы уже отмечали, из работы [7] следует, что $G_{2}$-многообразие $M$ обладает тремя невырожденными единичными векторными полями $\xi_{i}$. На $\xi_{i}^{\perp}$ можно ввести почти комплексную структуру по формуле (5).

В работе [8] показано, что если $M$ обладает метрикой с группой голономии, являющейся подгруппой $G_{2}$, то данные почти комплексные структуры интегрируемы.

Вследствие интегрируемости, мы выдвигаем следующую гипотезу.

ГиПотезА. Любое многообразие с группой голономии $G_{2}$ реализуется в виде удовле-

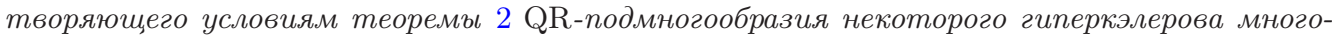
образия.

Автор благодарит И. А. Тайманова и Я. В. Базайкина за ценные замечания и поддержку. 


\section{СПИСОК ЦИТИРОВАННОЙ ЛИТЕРАТУРЫ}

[1] R. L. Bryant, S. M. Salamon, Duke Math. J., 58:3 (1989), 829-850. [2] D. D. Joyce, J. Differential Geom., 43:2 (1996), 291-328; D. D. Joyce, 329-375. [3] A. Kovalev, J. Reine Angew. Math., 565 (2003), 125-160. [4] A. Kovalev, J. Nordström, Ann. Global Anal. Geom., 38:3 (2010), 221-257. [5] A. Gray, Trans. Amer. Math. Soc., 141 (1969), 465-504; Trans. Amer. Math. Soc., 148:2 (1970), 625. [6] A. Bejancu, Proc. Amer. Math. Soc., 69:1 (1978), 135-142; Trans. Amer. Math. Soc., 250 (1979), 333-345. [7] E. Thomas, Ann. of Math. (2), 85:2 (1967), 184-217. [8] M. Verbitsky, A CR twistor space of a $G_{2}$-manifold, arXiv: 1003.3170.

Д. В. Егоров

Поступило

Северо-Восточный федеральный университет

28.04.2011

им. М. К. Аммосова, г. Якутск

E-mail: egorov.dima@gmail.com 